\title{
Dysregulated Sonic hedgehog signaling and medulloblastoma consequent to IFN- $\alpha$-stimulated STAT2-independent production of IFN- $\gamma$ in the brain
}

\author{
Jianping Wang, ${ }^{1}$ Ngan Pham-Mitchell, ${ }^{1}$ Christian Schindler, ${ }^{2}$ and Iain L. Campbell ${ }^{1}$
}

${ }^{1}$ Department of Neuropharmacology, The Scripps Research Institute, La Jolla, California, USA

${ }^{2}$ Department of Microbiology, Columbia University, New York, New York, USA

\begin{abstract}
The type I IFNs (IFN- $\alpha$ and IFN- $\beta$ ), which are crucial in antiviral defense and immune regulation, signal via the Janus kinase/signal transducer and activator of transcription (JAK/STAT) pathway with activation of STAT1 and STAT2. Here, the function of STAT2 was studied in transgenic mice (termed GIFN/STAT2 ${ }^{--}$) with CNS production of IFN- $\alpha$. Surprisingly, GIFN/STAT2 ${ }^{-/-}$, but not GIFN/STAT1null, transgenic mice, with CNS production of IFN- $\alpha$, died prematurely with medulloblastoma. An immune response also induced in the brain of the GIFN/STAT2 $2^{--}$mice was associated with IFN- $\gamma$ gene expression by $\mathrm{CD}^{+} \mathrm{T}$ cells and the activation of the STAT1, STAT3, STAT4, and STAT5 molecules. Expression of the Sonic hedgehog $(\mathrm{Sh} h)$ and the downstream transcriptional factor Gli-1 genes, implicated in the pathogenesis of medulloblastoma, was found to be significantly increased and cotranscribed in cerebellar granule neurons of the GIFN/STAT2 $2^{-/-}$mice. IFN- $\gamma$, but not IFN- $\alpha$, induced STAT1-dependent expression of the Shb gene in cultured cerebellar granule neurons. Thus, there is an unexpected and extraordinarily adverse biological potency of IFN- $\alpha$ in the CNS when the primary signal transduction molecule STAT2 is absent. Moreover, a hitherto unknown role is indicated for the immune system in the pathogenesis of developmental disorders and tumorigenesis of the CNS via dysregulated Shh signaling mediated by IFN- $\gamma$.
\end{abstract}

J. Clin. Invest. 112:535-543 (2003). doi:10.1172/JCI200318637.

\section{Introduction}

The type I IFNs (IFN- $\alpha$ and IFN- $\beta$ ) bind to a common receptor and are crucial effector cytokines in many antiviral, antiproliferative, and immune responses $(1,2)$. However, these cytokines are also implicated in the pathogenesis of infectious (3), genetic (4), and autoimmune (5) disorders. The cellular actions of the type I IFNs result primarily from their activation of the Janus kinase/signal transducer and activator of transcription (JAK/STAT) signaling pathway $(6,7)$. Type I IFN receptor (IFNAR) activation triggers the JAK-mediated tyrosine phosphorylation of STAT2 and STAT1. These activated STAT molecules form heterodimers that translocate

Received for publication April 14, 2003, and accepted in revised form June 24, 2003

Address correspondence to: Iain L. Campbell, The Scripps Research Institute, SP315, 10550 N. Torrey Pines Road, La Jolla, California 92037, USA. Phone: (858) 784-9306;

Fax: (858) 784-9544; E-mail: icamp@scripps.edu.

Conflict of interest: The authors have declared that no conflict of interest exists.

Nonstandard abbreviations used: Janus kinase (JAK); signal transducer and activator of transcription (STAT); type I IFN receptor (IFNAR); IFN regulatory factor (IRF); IFN-stimulated gene factor-3 (ISGF3); IFN-stimulated response element (ISRE); glial fibrillary acidic protein (GFAP); GFAP-IFN- $\alpha$ (GIFN); RNase protection assay (RPA); Sonic hedgehog (Shh); proliferative cell nuclear antigen (PCNA); external germinal layer (EGL); $\gamma$-activated sequence (GAS). to the nucleus and associate with an additional factor, IFN regulatory factor-9 (IRF-9), forming the complex termed IFN-stimulated gene factor-3 (ISGF3). ISGF3 then interacts with the IFN-stimulated response element (ISRE) to modulate the transcription of over 300 genes (8).

The importance of the JAK/STAT pathway in mediating the actions of the type I IFNs is underscored by the observations that mice lacking either STAT1 $(9$, 10) or STAT2 (11) have impaired type I IFN responses and are highly susceptible to viral infection. Nevertheless, other findings in STAT1-null mice showing that antiviral responses occur to type I IFNs (12), and that the neuropathogenic actions of IFN- $\alpha$ are markedly exacerbated (13), point to alternative, but as-yet unidentified, signaling pathways that can mediate both physiological and pathophysiological actions of these cytokines. STAT1 is a key factor not only in signal transduction coupled to IFNAR but also in that coupled to the type II IFN (i.e., IFN- $\gamma$ ) receptor (6). In addition, activation of STAT 1 can occur in response to other cytokines, such as IL-6 (14). By contrast, STAT2 is implicated in only IFNAR-coupled signal transduction (6). However, it is unknown whether type I IFNs can signal and alter any cellular functions in the absence of STAT2 and what the physiological consequences of this alternative signaling might be. To study these issues, here we developed glial fibrillary acidic protein-IFN- $\alpha(G F A P-I F N-\alpha$; hereafter called 
GIFN) transgenic mice with CNS-specific astrocyte production of IFN- $\alpha_{1}(15,16)$ that were null for the STAT2 gene (11).

\section{Methods}

Animals. Previously characterized GIFN transgenic

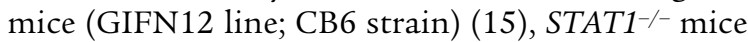
(9), and STAT2 ${ }^{-/-}$(11) mice (both 129/Sv background) were used. GIFNXSTAT null mice were produced by interbreeding of GIFN transgenic mice with the respective STAT-null mice, and the genotype of progeny was verified by PCR analysis of tail DNA. Handling of mice and experimental procedures were conducted in accordance with the NIH guidelines for animal care and use.

RNase protection assay. Total RNA and poly $(\mathrm{A})^{+} \mathrm{RNA}$ were isolated from freshly dissected snap-frozen brain or cerebellum using TRIZOL reagent (Life Technologies Inc., Gaithersburg, Maryland, USA) and oligo-dT (Ambion Inc., Austin, Texas, USA), respectively, as described previously (17). RNase protection assays (RPAs) were performed and RNA levels were quantified from autoradiographs by densitometry using NIH Image software (version 1.31) as described previously (18). RPA probes for Sonic hedgehog (Shh) and Gli-1 were cloned by PCR using cDNA templates generously provided by Matthew Scott (Stanford University, San Jose, California, USA) and Alexandra L. Joyner (The Skirball Institute, New York University School of Medicine, New York, New York, USA), respectively. The Patched1 (Ptch1) RPA probe was synthesized by RT-PCR using total RNA extracted from mouse brain as substrate and cloned as described previously (18). The targeted sequences for Shh, Gli-1, and Ptch1 riboprobes were 121-446 (GenBank X76290), 2041-2331 (GenBank NM_010296), and 3601-3831 (GenBank U46155), respectively. Other multiprobe sets for the different STATs, IFN-regulated genes, and IFNs were described previously $(17,18)$.

In situ hybridization and immunocytochemistry. Brains were removed, and one hemibrain was fixed overnight in ice-cold 4\% paraformaldehyde in PBS ( $\mathrm{pH}$ 7.4). Paraffin-embedded sagittal sections $(8 \mu \mathrm{m})$ were prepared for in situ hybridization. ${ }^{33}$ P-labeled cRNA probes transcribed from the linearized Shh, Gli-1, or IFN- $\gamma$ RPA plasmids described above were used for in situ hybridization performed as described previously $(17,18)$. For dual labeling, hybridized sections were incubated with the following antibodies: rabbit anti-human CD3 (DAKO Corp., Carpinteria, California, USA), rabbit anti-cow GFAP (DAKO Corp.), mouse anti-human neurofilament (Sternberger Monoclonals Inc., Lutherville, Maryland, USA), or biotinylated lectin from Lycopersicon esculentum (Sigma-Aldrich, St. Louis, Missouri, USA), to identify T lymphocytes, astrocytes, neurons, or cells of the monocytic lineage, respectively. Subsequent immunoperoxidase staining was performed using Vectastain $\mathrm{ABC}$ kits (Vector Laboratories Inc.,
Burlingame, California, USA) and 3',5'-diaminobenzidine $/ \mathrm{H}_{2} \mathrm{O}_{2}$ reagent as substrate. For additional immunohistochemistry, the other brain hemisphere was embedded in OCT compound (Sakura Finetek USA Inc., Torrance, California, USA) and snap-frozen. Sagittal sections $(10 \mu \mathrm{m})$ were cut with a cryostat microtome. Mouse anti-proliferative cell nuclear antigen (anti-PCNA) mAb (BD PharMingen, San Diego, California, USA) was used to label proliferating cells. The following rat mAb's were used for immunophenotyping of frozen sections: anti-mouse pan-leukocyte (CD45), anti-mouse $\mathrm{CD} 4$, and anti-mouse CD8, all from BD PharMingen. All antibodies were used at a final concentration of $5 \mu \mathrm{g} / \mathrm{ml}$ diluted in blocking buffer. Bound antibody was detected using Vectastain ABC kits (Vector Laboratories Inc.). Prior to mounting, sections were counterstained with Mayer's hematoxylin, and dehydrated in graded ethanols.

Western blot. Total-protein lysates of cerebellum were prepared and analyzed by SDS-PAGE followed by immunoblotting as described previously (13). Antibodies against Shh-N, Shh, STAT1, STAT4, STAT5, and actin were purchased from Santa Cruz Biotechnology Inc. (Santa Cruz, California, USA). Anti-phosphotyrosine-STAT1, -STAT3, and -STAT5 antibodies were obtained from Cell Signaling Technology Inc. (Beverly, Massachusetts, USA). AntiSTAT3 and anti-phosphotyrosine-STAT4 antibodies were obtained from BioSource International Inc. (Camarillo, California, USA) and Zymed Laboratories Inc. (South San Francisco, California, USA), respectively. Bound antibodies were visualized using peroxidase-conjugated secondary antibody and then detected using an ECL kit (Pierce Chemical Co., Rockford, Illinois, USA). The membrane was stripped in buffer (62.5 mM Tris-HCl, $\mathrm{pH}$ 6.8, with $2 \%$ SDS and $100 \mathrm{mM} \beta$-mercaptoethanol) for 30 minutes at $50^{\circ} \mathrm{C}$ before reblotting.

Granule neuron culture. Cerebellum from postnatal day $5 \mathrm{WT}$, STAT1 ${ }^{-/-}$, or STAT2-/- mice was dissected and digested at $37^{\circ} \mathrm{C}$ for 10 minutes in HBSS with $0.25 \%$ trypsin without $\mathrm{Ca}^{++}$and $\mathrm{Mg}^{++}$(Invitrogen Corp., San Diego, California, USA). The digestion mixture was then treated with 0.004\% DNase (Roche Diagnostics Corp., Indianapolis, Indiana, USA) and $0.03 \%$ soybean trypsin inhibitor (Sigma-Aldrich). After trituration with a fire-polished Pasteur pipette and washing with HBSS, the single-cell suspension was resuspended in Neurobasal medium with $2 \%$ FCS and 1\% B-27 supplement (all from Invitrogen Corp.), counted by trypan blue exclusion analysis, and plated on a $75-\mathrm{ml}$ culture flask at a density of $9.0 \times 10^{5}$ cells $/ \mathrm{ml}$. After an initial 48 hours of incubation $\left(5 \% \mathrm{CO}_{2}\right.$ at $\left.37^{\circ} \mathrm{C}\right)$, the culture medium was replaced with the same medium but no serum and incubated for another 24 hours. Finally, murine IFN- $\alpha$ $(250 \mathrm{IU} / \mathrm{ml}$; Roche Diagnostics Corp.) or IFN- $\gamma(50$ $\mathrm{IU} / \mathrm{ml} ; \mathrm{R} \& \mathrm{D}$ Systems Inc., Minneapolis, Minnesota, 


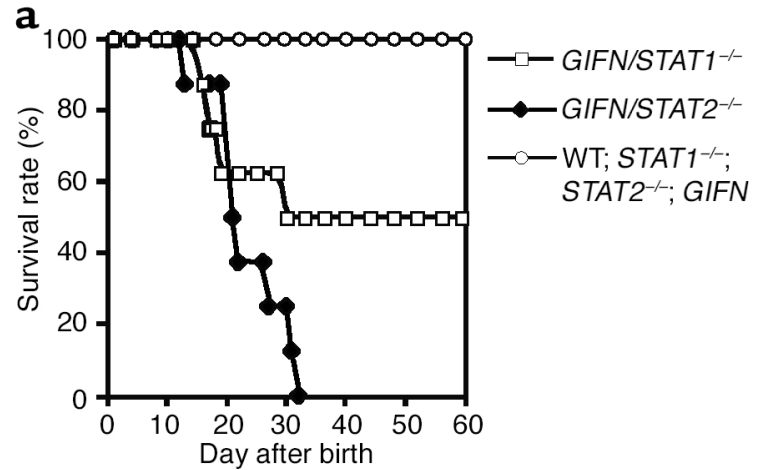

b

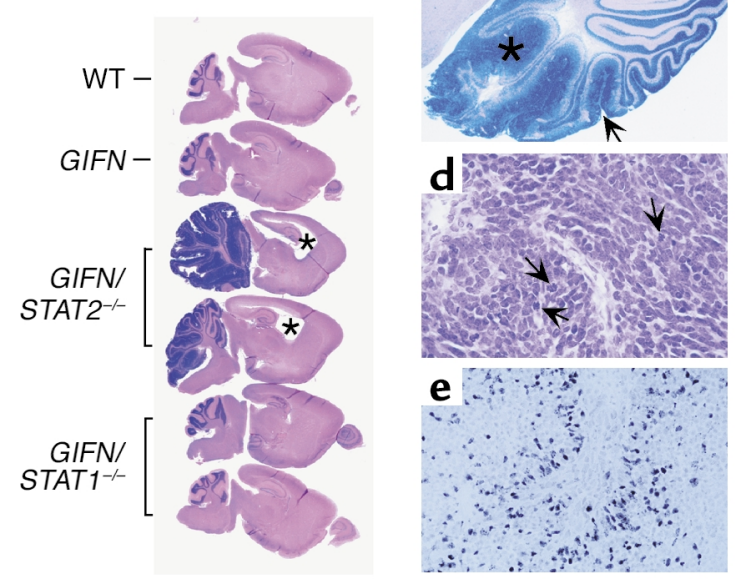

Figure 1

GIFN mice lacking STAT2 die prematurely with medulloblastoma. (a) Survival curves for GIFN/STAT2 ${ }^{-/-}$mice compared with GIFN/STAT1-/- mice and other control mice $(n=8-10$ mice per group). (b) Gross pathology of the brain. Sagittal brain sections (10 $\mu \mathrm{m}$ ) stained with $\mathrm{H} \& \mathrm{E}$, showing considerable enlargement of the cerebellum due to diffusely spreading medulloblastoma and hydrocephalus (asterisks) in 3-week-old GIFN/STAT2 ${ }^{-1-}$ mice compared with age-matched WT, GIFN, and GIFN/STAT1 ${ }^{-1-}$ mice. (c) Sagittal section $(10 \mu \mathrm{m})$ through the cerebellum of a 3-week-old GIFN/STAT1-/- mouse, showing abnormal presence and hyperplasia of the external germinal layer (arrow) and early tumor formation (asterisk). (d) $\mathrm{H} \& \mathrm{E}$-stained section through a medulloblastoma in the cerebellum of a GIFN/STAT2-/- mouse. The tumors were composed of small, darkly stained cells with a sparse cytoplasm and prominent nucleus. Numerous mitotic figures were evident (arrows). (e) Sagittal frozen section $(10 \mu \mathrm{m})$ through a medulloblastoma from a GIFN/STAT2 ${ }^{-/-}$mouse, immunostained for PCNA. Original magnifications: $\times 1$ for $\mathbf{b} ; \times 4$ for $\mathbf{c} ; \times 600$ for $\mathbf{d} ; \times 400$ for $\mathbf{e}$.

USA) was added to the culture and incubated for 4 hours at $37^{\circ} \mathrm{C}$. Thereafter, the total RNA was extracted using TRIZOL reagent.

Splenic-leukocyte analysis. Erythrocyte-free spleen cell suspensions were prepared from 2- to 3-month-

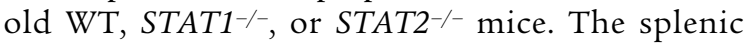
leukocytes $\left(2 \times 10^{7}\right.$ viable cells) were resuspended in RPMI-1640 medium (Invitrogen Corp.) containing $1 \%$ BSA with or without murine IFN- $\alpha(500 \mathrm{IU} / \mathrm{ml}$; Roche Diagnostics Corp.) and placed at $37^{\circ} \mathrm{C}$ for 2 hours. Thereafter, total RNA was extracted using TRIZOL reagent. For immunoblot analysis, splenic leukocytes were treated with IFN- $\alpha$ for 30 minutes, and total-protein lysates were prepared and analyzed as described above.

Statistical analysis. All data are given as the mean \pm SEM. Data were analyzed by two-tailed unpaired Student's $t$ test with a significance level of 0.05 .

\section{Results}

Increased lethality and medulloblastoma in GIFN mice with STAT2 deficiency. While control mice (WT, STAT2-/, $S T A T 1^{-1-}$, and GIFN) appeared healthy, GIFN mice null for STAT2 (denoted GIFN/STAT2--) failed to thrive from birth, had ataxia, and nearly all died by 1 month of age (Figure 1a). Consistent with our previous report (13), GIFN/STAT1 ${ }^{-/-}$mice also had an increased lethality and disease phenotype, but this was less severe than in the GIFN/STAT2 ${ }^{--}$animals and was associated with neurodegenerative disease affecting the ventromedial forebrain. Histological examination of the brains of 3-week-old GIFN/STAT2 $2^{--}$ mice revealed, in addition to hydrocephalus, gross enlargement of the cerebellum with a loss of cellular architecture due to diffuse tumor growth (Figure 1b). In less advanced cases, abnormal persistence and marked hyperplasia of the external germinal layer (EGL) of the cerebellum was seen (Figure 1c, arrow). Typically, and as was observed here in control and GIFN/STAT1 $1^{-/-}$mice, the EGL disappears by postnatal day 14 (19). The tumors in the GIFN/STAT2-/mice appeared to originate from the abnormal EGL and resembled those of human medulloblastoma (20), being composed of small dark-blue-staining cells with a prominent nucleus and a sparse cytoplasm that appeared relatively undifferentiated (Figure 1, c and d). Extensive proliferation of these cells was seen throughout the tumors and in the EGL, as evidenced by the presence of many mitotic figures (Figure 1d, arrows) and of numerous cells positive for PCNA (Figure 1e). In all, these findings revealed catastrophic consequences of STAT2-independent IFN- $\alpha$ actions in the CNS of mice, namely, the development of medulloblastoma and early death.

Significantly increased activation of various STATs and expression of IFN- $\gamma$-regulated genes in the brains of GIFN/STAT2 $/-$ mice. The finding of medulloblastoma in the GIFN/STAT2 ${ }^{-/-}$mice suggested that the activation of alternative signaling pathways might mediate novel tumorigenic actions of IFN- $\alpha$ in the developing brain. Candidates included STAT1 (11), STAT3 $(21,22)$, and STAT5 (23), which have all been shown to be activated by IFN- $\alpha$. We therefore examined the levels of these molecules in the brain. The steady-state levels of STAT1 protein were increased markedly in the cerebellum of GIFN and GIFN/ STAT2 ${ }^{-/}$mice (Figure 2a). However, additionally in the GIFN/STAT2 $2^{-/-}$mice, phosphotyrosine-STAT1, phosphotyrosine-STAT3, and phosphotyrosineSTAT5 levels were also increased. Therefore, despite STAT2 deficiency, STAT1, STAT3, and STAT5 were 
all activated and STAT1 levels strongly upregulated in the presence of IFN- $\alpha$ in the cerebellum of the GIFN/STAT2-/- mice.

In addition to heterodimeric association with STAT2, STAT1 also forms homodimers that translocate to the nucleus and bind to the DNA recognition element, known as the $\gamma$-activated sequence (GAS), which is essential for IFN- $\gamma$ regulation of gene transcription $(6,7)$. To determine whether activation of STAT 1 found in the cerebellum of the GIFN/STAT2 ${ }^{-/-}$mice resulted in functional signaling, we investigated the expression of a number of known ISRE- and GAS-driven genes (6, 7 ). In contrast to GIFN mice, which had increased expression of the ISRE-driven genes RNA-dependent protein kinase $R(P K R)$ and 2',5',oligoadenylate synthetase (OAS) (Figure 2b, upper panel), GIFN/STAT2 ${ }^{-/-}$ mice showed reduced expression of these genes and a significant shift to the expression of the GASdriven genes class II trans-activator (CIITA), IFN-regulated factor-1 (IRF1), and Tlymphocyte GTPase (TGTP) (Figure 2b, lower panel). Thus, the activation of STAT1 in the cerebellum of the GIFN/STAT2-/mice leads to functional signaling characteristic of STAT1 homodimers with the induction of GASdriven gene expression.
STAT2-independent, IFN- $\alpha$-stimulated IFN- $\gamma$ gene expression and activation of STAT4 in the brain and splenic leukocytes. The activation of STAT1 and downstream GAS-driven gene expression in the cerebellum of the GIFN/STAT2 ${ }^{-/-}$mice suggested the possible involvement of IFN- $\gamma$, which is a primary modulator of these processes $(6,7)$. We therefore examined the expression of IFN- $\gamma$ and the transgene-encoded IFN- $\alpha 1$ genes in both hindbrain and forebrain. Compared with GIFN mice, and consistent with a STAT1 dependence of the GFAP transgene promoter, IFN- $\alpha_{1}$ mRNA levels were significantly altered, being increased in GIFN/STAT2 $2^{--}$and decreased in GIFN/STAT1 ${ }^{-/-}$mice (Figure 3a). No IFN- $\alpha_{1}$ mRNA was detectable in WT or STAT-null controls. IFN- $\gamma$ mRNA was detected readily in both brain regions in GIFN/STAT2 $2^{-/-}$mice and at very low levels in GIFN and GIFN/STAT1 ${ }^{-1-}$ mice, but not detected in WT and STAT-null controls (Figure 3a). Since IFN- $\gamma$ is produced primarily by activated $T$ cells and NK cells, we asked whether there was an immune pathology in the brain of the GIFN/STAT2 ${ }^{-/}$mice. Compared with WT (not shown) and GIFN mice (Figure 3b), markedly increased numbers of cells positive for the pan-leukocyte marker CD45 were located around blood vessels and scattered throughout the tumor (Figure 3c) as well a

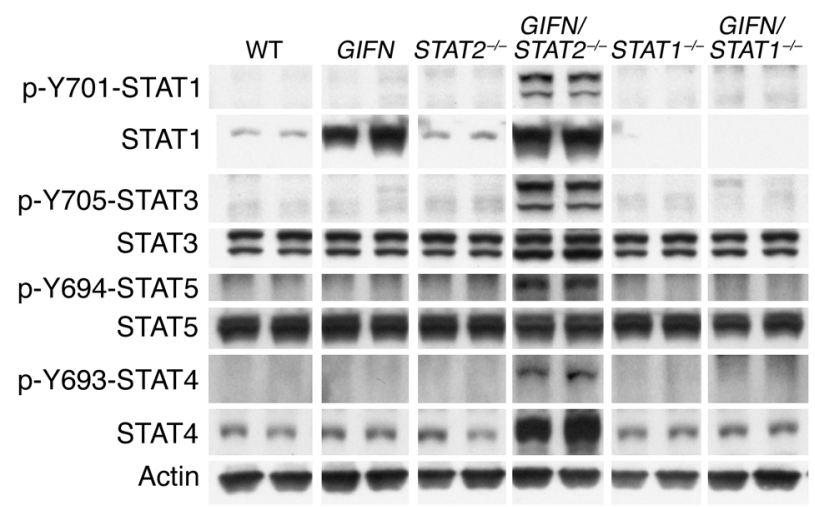

b

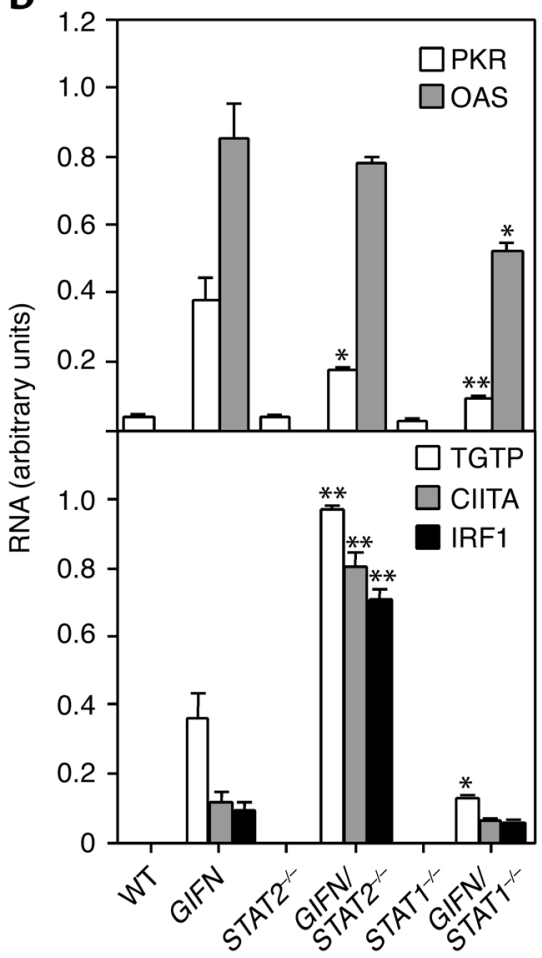

Figure 2

Activation of STAT molecules and GAS-driven gene expression in the cerebellum of GIFN/STAT2 ${ }^{-1-}$ mice. (a) Total-protein lysates (100 $\mu \mathrm{g}$ per sample) from the cerebellum of 3-week-old mice were immunoblotted with anti-phosphotyrosine-STAT1, -STAT3, -STAT4, or -STAT5 antibody. The filters were stripped and reblotted with anti-STAT and anti-actin (sample loading control) antibodies as shown. (b) Analysis of ISRE-driven (upper panel) and GAS-driven (lower panel) gene expression. Total RNA (5 $\mu \mathrm{g}$ ) from the cerebellum of 3-week-old mice was analyzed by RPA, and the level of mRNA transcripts was quantitated as described previously $(17)$. ${ }^{*} P<0.05$ and ${ }^{*} P<0.01$ in GIFN/STAT2 ${ }^{--}(n=4)$ compared with GIFN mice and other genotypes $(n=4-5)$. 

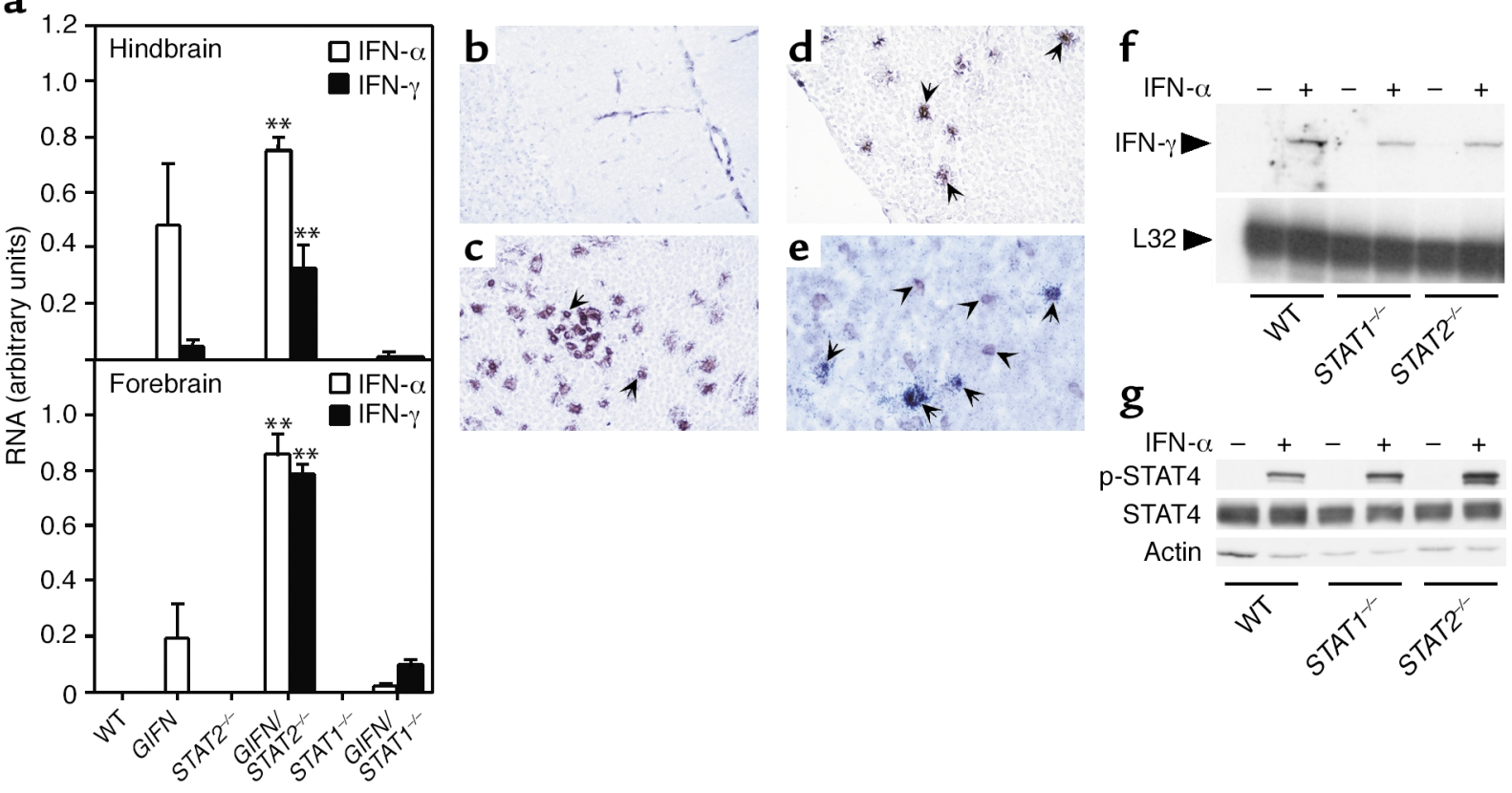

\section{Figure 3}

Immune pathology in the cerebellum of GIFN/STAT2 ${ }^{-/-}$mice and induction of IFN- $\gamma$ gene expression and activation of STAT4 in splenic leukocytes. (a) Analysis and quantitation of IFN gene expression by RPA, performed as described above for Figure $2 \mathrm{~b}$. ${ }^{*} P<0.01$ in GIFN/STAT2-/- $(n=4)$ compared with GIFN mice and other genotypes $(n=4-5)$. (b-d) Frozen brain sections $(10 \mu \mathrm{m})$ from 3-week-old GIFN (b) or GIFN/STAT2-/- (c and d) mice immunostained for CD45 (b and c; arrows) and CD4 (d; arrows). Original magnification: $\times 400$. (e) Dual in situ hybridization of IFN- $\gamma$ RNA transcripts and immunohistochemical localization of CD3 (arrows) in the cerebellum of 3-week-old GIFN/STAT2 ${ }^{-/-}$mice. CD3 ${ }^{+}$cells not expressing detectable IFN- $\gamma$ RNA are also shown (arrowheads). Original magnification: $\times 600$. (f) IFN- $\gamma$ gene expression by splenic leukocytes. Freshly isolated splenic leukocytes from the spleens of adult mice were treated with IFN- $\alpha(500 \mathrm{U} / \mathrm{ml})$ for 2 hours at $37^{\circ} \mathrm{C}$. Total RNA was then prepared and analyzed by RPA. (g) Immunoblot analysis for total and phosphotyrosine-STAT4 in total-protein lysates from IFN- $\alpha$-treated $\left(500 \mathrm{U} / \mathrm{ml}\right.$ for 30 minutes at $\left.37^{\circ} \mathrm{C}\right)$ splenic leukocytes prepared from mice of the different genotypes shown. Both $\mathbf{f}$ and $\mathbf{g}$ show representative results from three independent experiments.

as other brain regions such as the forebrain in GIFN/ $S T A T 2^{-/-}$mice (Figure 3c). Further analysis revealed the presence of $\mathrm{CD}^{+}$(Figure $3 \mathrm{~d}$, arrows) and $\mathrm{CD}^{+}$ $T$ cells (not shown), in similar numbers and distribution, peppered throughout the tumor as well as the rest of the brain. We next sought to determine the cellular source and localization of IFN- $\gamma$ gene expression in the brain. By dual-label in situ hybridization analysis, IFN- $\gamma$ RNA was detectable in the brains of GIFN/STAT2 $2^{-\prime-}$ mice only. The IFN- $\gamma-$ expressing cells, like the $T$ cells, were scattered throughout the brain and tumor, and, consistent with production by the $T$ cells, they were colocalized almost exclusively with $\mathrm{CD}^{+}$cells (Figure $3 e$, arrows). These observations indicated that a generalized immune pathology was present in the brain of the GIFN/ $S T A T 2^{-/-}$mice, with activated $\mathrm{T}$ cells being responsible for the IFN- $\gamma$ gene expression.

IFN- $\alpha$ can induce IFN- $\gamma$ production by splenic leukocytes from WT and STAT1-null mice via the activation of STAT4 $(24,25)$. We asked whether a similar response could occur in splenic leukocytes lacking STAT2 and thus provide a mechanism for the IFN- $\gamma$ induction seen in the brain of the GIFN/STAT2 ${ }^{-/-}$ mice. We found that, compared with untreated splenic leukocytes, in splenic leukocytes from WT,
STAT1 ${ }^{-/}$, and $S T A T 2^{-/-}$mice, IFN- $\alpha$ induced both IFN- $\gamma$ mRNA expression (Figure $3 \mathrm{f}$ ) and phosphotyrosine-activated STAT4 (Figure 3g). Furthermore, a survey of STAT4 in the cerebellum of GIFN/STAT2 ${ }^{-/-}$ mice revealed that both steady-state and phosphotyrosine-activated STAT4 levels were induced (Figure $2 a)$. These findings not only confirmed that IFN- $\alpha$ can activate STAT4 and induce the expression of the IFN- $\gamma$ gene by murine splenic leukocytes but also indicated that this process clearly does not require STAT2; and they established that activation of STAT4 also occurred in vivo in the brain of GIFN/STAT2 ${ }^{-/-}$mice, further implicating this signaling pathway in IFN- $\alpha-$ mediated IFN- $\gamma$ gene expression.

Marked activation of the Shb pathway in GIFN mice with STAT2 deficiency. The Shh signaling pathway is essential for the development of the cerebellum and is also implicated in the initiation of medulloblastoma (26, 27). During development of the cerebellum, Shh produced by Purkinje neurons binds to the receptor Patched1 (Ptch1) on EGL granule neurons, derepressing the membrane protein Smoothened and activating the transcriptional factor Gli-1, resulting in the induction of gene expression and proliferation of the EGL neurons $(26,27)$. We therefore asked whether dysregulation of the Shh pathway might 
underlie the development of medulloblastoma in the GIFN/STAT2 ${ }^{-/-}$mice. The expression of the Shb and Gli-1 genes, but not Ptch1, was significantly increased in cerebellum from GIFN/STAT2 $2^{-/}$mice compared with control and GIFN/STAT1 ${ }^{-/-}$mice (Figure 4a). Shh is synthesized as a precursor molecule that undergoes autocleavage to yield an active $\mathrm{N}$-terminal fragment and a catalytic C-terminal fragment $(28,29)$. In the cerebellum of the GIFN/STAT2-/mice, levels of the N-terminal Shh fragment were increased, while the Shh precursor was decreased, consistent with increased secretion and processing of the Shh molecule (Figure 4b). Surprisingly, in situ hybridization analysis showed that the upregulated Shh and Gli-1 transcripts were colocalized in EGL granule neurons and the medulloblastoma cells (Figure $4 \mathrm{c}$ ). In conclusion, the development of medulloblastoma in the GIFN/STAT2 ${ }^{-/-}$mice coincided with increased activation of the Shh signaling pathway and, furthermore, the persistent ectopic expression of Shh by the EGL cells.

The mechanisms that control Shb gene transcription in the EGL granule neurons or Purkinje neurons are unknown. We asked whether IFN- $\alpha$ or IFN- $\gamma$ could fulfill such a role. Cultured granule neurons from WT and STAT2 ${ }^{-/-}$mice treated with either IFN- $\alpha$ or IFN- $\gamma$ had significantly increased STAT1 mRNA levels (Figure 4d). These findings indicate not only that the IFNs act on granule neurons but also that IFN- $\alpha$ can regulate the expression of some genes (e.g., STAT1) in these cells via a STAT2-independent pathway. However, in contrast to STAT1 expression, Shh gene expression was induced by IFN- $\gamma$, but not IFN- $\alpha$, in neurons from WT and $S T A T 2^{--}$, but not STAT1 ${ }^{--}$, mice (Figure $4 \mathrm{~d}$ ). Thus, IFN- $\gamma$ is a novel STAT1-dependent inducer of the $S h b$ gene in granule neurons.

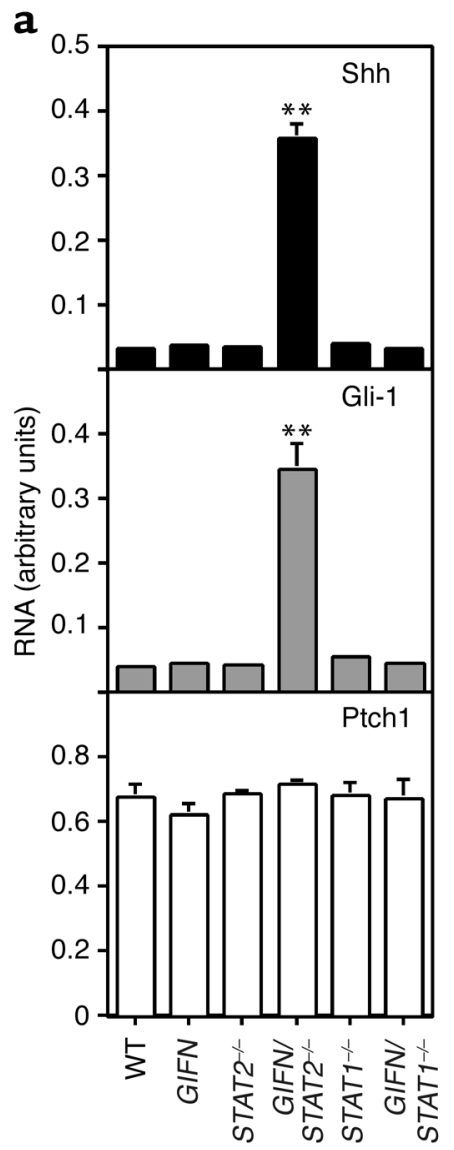

C
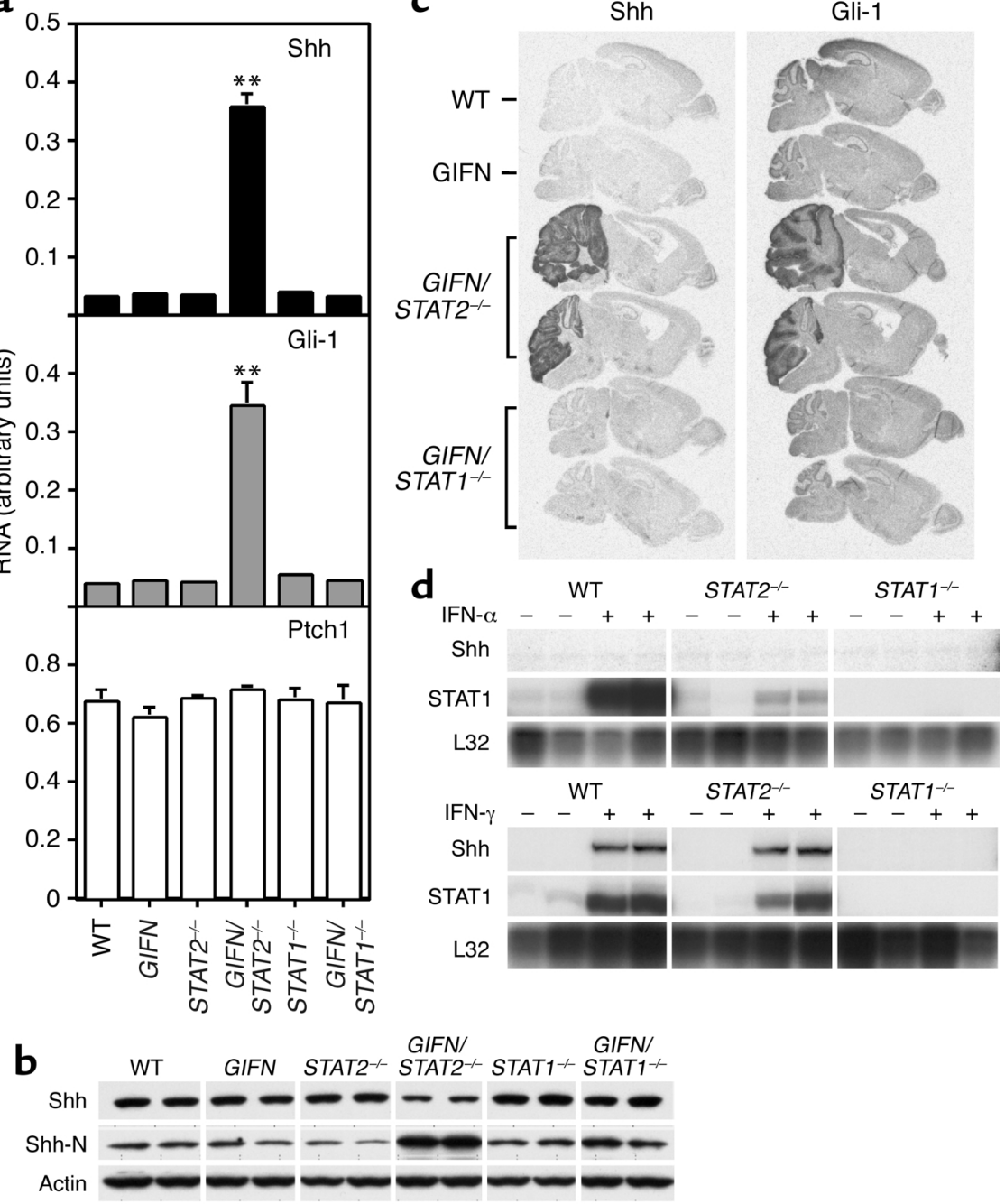

\section{Figure 4}

Activation of the Shh signaling pathway. (a) RNase protection analysis of Shh, Gli-1, and Ptch1 mRNAs $\left(n=5-7,{ }^{*} P<0.01\right.$ compared with control groups). (b) Total-protein lysates ( $100 \mu \mathrm{g}$ per sample) from the cerebellum of 3 -week-old mice were immunoblotted with anti-Shh, anti-Shh-N, and anti-actin (sample loading control) antibodies. (c) Localization by in situ hybridization of Shh and Gli-1 mRNA transcripts in the cerebellum of 3-week-old mice. (d) Expression of the Shh and STAT1 genes by cultured granule neurons. Cells were exposed to either IFN- $\alpha$ (upper panel) or IFN- $\gamma$ (lower panel) for 4 hours at $37^{\circ} \mathrm{C}$. Total RNA was then prepared and analyzed by RPA. 


\section{Discussion}

Activated STAT1 and STAT2 form heterodimers that, together with IRF-9, combine to make the transcriptionally active complex ISGF3, which is responsible for mediating many key actions of the type I IFNs. However, recent studies in STAT1-null mice and cells indicate that type I IFNs maintain some biological and pathobiological functions, pointing to the existence of alternative IFNAR-coupled signaling pathways $(12,13,24)$. We now show that despite the absence of STAT2, a primary signaling molecule linked to IFNAR activation, IFN- $\alpha$ in the CNS of mice induces an unexpectedly profound neurological disorder characterized by medulloblastoma, inflammation, and early death. Although GIFN mice deficient in STAT1 also have an accelerated and more severe neurological disease phenotype, the pathological hallmarks of this disorder are quite distinct, consisting of calcification and neurodegeneraton (13). Thus, not only do our findings reveal an extraordinarily adverse biological potency of IFN- $\alpha$ in the CNS when either of the primary signal transduction molecules STAT1 and STAT2 is deficient, but they highlight the point that fundamentally unique roles may exist for these transcriptional factors in the cellular response following IFNAR activation. A further possibility is that alternative signaling pathways are engaged by the IFNAR that are independent of both STAT1 and STAT2. Recent published reports, together with our studies described here, offer support for all three mechanisms. Thus, IFN- $\alpha$ can stimulate both (a) the STAT1-independent nuclear translocation of STAT2, which binds to Brg-1, forming a transcriptionally active complex that can induce the expression of specific genes (30), and (b) the STAT2-independent activation (11) and increased expression (see Figure 4d) of STAT1. Finally, IFN- $\alpha$ activation of the unique transcriptional factor STAT4 is both STAT1 independent (25) and STAT2 independent (see Figure 3g).

Despite initial findings to the contrary (31-33), it is now evident that in mice, like in humans, IFN- $\alpha$ has a significant role in promoting the development of the Th1 immune response, bridging innate and adaptive immunity $(24,25)$. Consistent with this role, IFN- $\alpha$, in concert with $\mathrm{T}$ cell receptor signaling, induces IFN- $\gamma$ production by $\mathrm{T}$ cells - an effect mediated by direct IFN- $\alpha$ activation of STAT4 (25). Although these actions of IFN- $\alpha$ are STAT1 independent $(24,25)$, whether they might also occur in the absence of STAT2 was unclear and had been questioned (33). However, our findings clearly showed that, both in the brain and in splenic leukocytes, IFN- $\alpha$ stimulated the activation of STAT 4 and IFN- $\gamma$ gene expression in the absence of STAT2. Moreover, our findings indicated that the induction of IFN- $\gamma$ gene expression in the brain under these circumstances was due to infiltrating $\mathrm{CD}^{+} \mathrm{T}$ cells. Thus, the ability of IFN- $\alpha$ to promote Th1 immune responses is not compromised but enhanced by the absence of STAT2.
In addition to STAT1 (11), other signal transduction molecules can be activated in certain cell types by type I IFNs. These include the STAT transcriptional factors STAT3 $(21,22)$ and STAT5 $(23)$. Whether the activation of alternative signaling pathways involving activated STAT3 and STAT5 in response to IFN requires STAT2 is unclear. Here we found that both STAT3 and STAT5 were activated in the cerebellum of GIFN mice deficient in STAT2. Therefore, one possibility is that the activation of STAT3 and STAT5 in the brains of the GIFN/STAT2-null mice is mediated directly by the action of IFN- $\alpha$ on target cells. However, this interpretation is complicated by the complex immunopathological and tumorigenic phenotype of these mice. Further studies are now required to determine the regulation, distribution, and cellular localization of the activated STAT3 and STAT5 in the brain of the GIFN/STAT2-null mice. Moreover, the biological consequences of STAT3 and STAT5 activation in the CNS remain unknown, although it is tempting to speculate that either or both of these molecules might contribute to the development of medulloblastoma. Thus, in the case of both STAT3 and STAT5, constitutive activation has been demonstrated in a wide range of human primary tumors (34), including medulloblastoma (35), while STAT3 activation is required for oncogenic transformation of some cell lines in vitro (34).

The unexpected development of medulloblastoma in the GIFN mice null for STAT2 was linked to a significant increase in the activity of the developmental signaling pathway involving Shh. During normal development, Shh produced by Purkinje neurons stimulates the proliferation of granule neuron progenitor cells in the EGL (36-38). Shh signaling induces the activation and increased gene transcription of Gli-1 (39). Dysregulation of this pathway, for example, via mutations producing deficiency in Patch 1 in mice and humans (26) or by overexpression of Shh in the mouse brain (40), predisposes to the development of medulloblastoma. Although the Shb gene is expressed transiently by early EGL neurons in the cerebellum of mice at postnatal day 2 (36), our findings revealed persistent expression of this gene by EGL neurons in the brains of GIFN mice null for STAT2. The increased expression of Gli-1 correlated closely with that of Shh, consistent with active signaling by the Shh pathway in these cells. These findings suggest a novel mechanism whereby sustained proliferation of the EGL neurons could occur in response to autocrine growth stimulation by Shh, eventually leading to their neoplastic transformation, giving rise to medulloblastoma.

The identity of factors that control the transcription of the Shb gene remains largely unknown. However, our finding that ectopic expression of Shh occurred in the presence of a strong inflammatory process in the cerebellum of GIFN mice null for STAT2 suggested that factors involved in this process 
might also be responsible for regulating Shb gene expression. Our studies identified one factor, IFN- $\gamma$, as the most likely candidate, since this cytokine was capable of inducing $S h b$ gene expression by cultured cerebellar granule neurons. Interestingly, like IFN- $\gamma$, IFN- $\alpha$ upregulated STAT1 gene expression by these cells, but did not influence $S h b$ gene expression, indicating that the effect of IFN- $\gamma$ was specific and likely mediated by GAS-responsive elements within the Shb gene promoter. Previous studies in transgenic mice with CNS-targeted IFN- $\gamma$ production found abnormal persistence and dysplasia of the EGL, although medulloblastoma was not found $(41,42)$. These studies offer additional support for a role of IFN- $\gamma$ in perturbing cerebellar EGL development, which, based on our findings here, we suggest occurs via the direct modulation of Shh signaling by this cytokine. However, further progression to medulloblastoma might depend on the loss of STAT2, which could function as a tumor repressor (30) and/or involve other mechanisms such as activation of STAT3- and STAT5dependent signaling (see above).

Our findings raise the question of their relevance to the pathogenesis of human neurological disease. A role for viruses in the etiology of human medulloblastoma is suspected $(43,44)$. It is conceivable that an antiviral immune response directed against such a viral infection might lead to IFN- $\gamma$ production in the brain during crucial developmental stages that expose the EGL to this cytokine. Also, various agents are known to inhibit or deplete STAT2 and/or STAT1, possibly producing dysregulated IFN signaling and altered biological responses. These include (a) viruses that have the capacity to block the activation or induction of these STATs by IFNs (45-47); (b) physiological inhibitors of STATS, e.g., the suppressors of cytokine signaling (SOCS) (48); and (c) the recently identified germline mutations in human STAT1 that cause defective IFN signaling $(49,50)$. This last point raises the possibility that mutations that similarly affect STAT2 might exist in the human population.

In conclusion, we have identified alternative IFN- $\alpha-$ signaling pathways in the brain that provoke a STAT2-independent, type I-like immune response with localized IFN- $\gamma$ production. We propose that IFN- $\gamma$ mediates the STAT1-dependent induction of Shh in early granule neurons of the EGL, resulting in the upregulation of the transcriptional factor Gli-1 and the perpetual growth and eventual neoplastic transformation of these cells to medulloblastoma. In addition to medulloblastoma, a highly malignant tumor with unknown etiology and pathophysiology that accounts for almost $20 \%$ of all brain tumors in children $(51,52)$, abnormal Shh signaling is found in a number of other human developmental tumors, including rhabdomyosarcoma and basal cell carcinoma $(26,27)$. Therefore, our study points to a hitherto unknown role for the immune system in the patho- genesis of developmental disorders and tumorigenesis of the CNS and perhaps other organs, involving dysregulated Shh signaling mediated by IFN- $\gamma$.

\section{Acknowledgments}

We thank Heather J. Weinkauf and Mary Wagner for their technical support, Matthew P. Scott (Stanford University) and Alexandra L. Joyner (New York University School of Medicine) for providing cDNA clones for murine Shh and Gli-1, respectively, and Robert Schreiber (Washington University School of Medicine) for providing STAT1 $1^{-/}$mice. We are grateful to Henry C. Powell and Eliezer Masliah (University of California at San Diego) for helpful discussion. This study was supported by NIH grants MH-62231 (to I.L. Campbell), MH-62261 (to I.L. Campbell), and GM-54686 (to C. Schindler) and National Multiple Sclerosis Society Research Grant RG 3237-A-1 (to C. Schindler). This is manuscript 15449-NP from The Scripps Research Institute.

1.Sen, G.C., and Ransohoff, R.M. 1993. Interferon-induced antiviral actions and their regulation. Adv. Virus Res. 42:57-102.

2. Belardelli, F., and Gresser, I. 1996. The neglected role of type 1 interferon in the T-cell response: implications for its clinical use. Immunol. Today. 17:369-372.

3. Krivine, A., et al. 1999. Measuring HIV-1 RNA and interferon- $\alpha$ in the cerebrospinal fluid of AIDS patients: insights into the pathogenesis of AIDS Dementia Complex. J. Neurovirol. 5:500-506.

4. Lebon, P., et al. 1988. Intrathecal synthesis of interferon-alpha in infants with progressive familial encephalopathy. J. Neurol. Sci. 84:201-208.

5. Shiozawa, S., Kuroki, Y., Kim, M., Hirohata, S., and Ogino, T. 1992. Interferon- $\alpha$ in lupus psychosis. Arthritis Rheum. 35:417-422.

6. Stark, G.R., Kerr, I.M., Williams, B.R.G., Silverman, R.H., and Schreiber, R.D. 1998. How cells respond to interferons. Annu. Rev. Biochem. 67:227-264.

7. Schindler, C., and Brutsaert, S. 1999. Interferons as a paradigm for cytokine signal transduction. Cell. Mol. Life Sci. 55:1509-1522.

8. Der, S.D., Zhou, A., Williams, B.R.G., and Silverman, R.H. 1998. Identification of genes differentially regulated by interferon $\alpha, \beta$, or $\gamma$ using oligonucleotide arrays. Proc. Natl. Acad. Sci. U. S. A. 95:15623-15628.

9. Meraz, M.A., et al. 1996. Targeted disruption of the Stat1 gene in mice reveals unexpected physiologic specificity in the JAK-STAT signaling pathway. Cell. 84:431-442.

10. Durbin, J.E., Hackenmiller, R., Simon, M.C., and Levy, D.E. 1996. Targeted disruption of the mouse Stat1 gene results in compromised innate immunity to viral disease. Cell. 84:443-450.

11. Park, C., Li, S., Cha, E., and Schindler, C. 2000. Immune response in Stat2 knockout mice. Immunity. 13:795-804

12. Gil, M.P., et al. 2001. Biologic consequences of Stat1-independent IFN signaling. Proc. Natl. Acad. Sci. U. S. A. 98:6680-6685.

13. Wang, J., Schreiber, R.D., and Campbell, I.L. 2002. STAT1 deficiency unexpectedly and markedly exacerbates the pathophysiological actions of IFN- $\alpha$ in the central nervous system. Proc. Natl. Acad. Sci. U. S. A. 99:16209-16214.

14. Schindler, C., and Strehlow, I. 2000. Cytokines and STAT signaling. Adv. Pharmacol. 47:113-174.

15. Akwa, Y., et al. 1998. Transgenic expression of IFN- $\alpha$ in the central nervous system of mice protects against lethal neurotropic viral infection but induces inflammation and neurodegeneration. J. Immunol. 161:5016-5026.

16. Campbell, I.L., et al. 1999. Structural and functional neuropathology in transgenic mice with CNS expression of IFN- $\alpha$. Brain Res. 835:46-61.

17. Asensio, V.C., Kincaid, C., and Campbell, I.L. 1999. Chemokines and the inflammatory response to viral infection in the central nervous system with a focus on lymphocytic choriomeningitis virus. J. Neurovirol. 5:65-75.

18. Maier, J., Kincaid, C., Pagenstecher, A., and Campbell, I.L. 2002. Regulation of signal transducer and activator of transcription and suppressor of cytokine signaling gene expression in the brain of mice with astrocyte-targeted production of IL-12 and experimental autoimmune encephalomyelitis. Am. J. Pathol. 160:271-288.

19. Miale, I., and Sidman, R.L. 1961. An autoradiographic analysis of histogenesis in the mouse cerebellum. Exp. Neurol. 4:277-296. 
20. Kleihues, P., et al. 2002. The WHO classification of tumors of the nervous system. J. Neuropathol. Exp. Neurol. 61:215-225.

21. Yang, C.-H., Murti, A., and Pfeffer, L.M. 1998. STAT3 complements defects in an interferon-resistant cell line: evidence for an essential role for STAT3 in interferon signaling and biological activities. Proc. Natl. Acad. Sci. U. S. A. 95:5568-5572.

22. Rani, M.R.S., et al. 1999. Catalytically active TYK2 is essential for interferon- $\beta$-mediated phosphorylatin of STAT 3 and interferon- $\alpha$ receptor-1 (IFNAR-1) but not for activation of phosphoinositol 3-kinase. J. Biol. Chem. 274:32507-32511.

23. Fasler-Kan, E., et al. 1998. Interferon- $\alpha$ activates signal transducers and activators of transcription 5 and 6 in Daudi cells. Eur. J. Biochem. 254:514-519.

24. Nguyen, K.B., et al. 2000 . Interferon $\alpha / \beta$-mediated inhibition and promotion of interferon- $\gamma$ : STAT1 resolves a paradox. Nat. Immunol. 1:70-76.

25. Nguyen, K.B., et al. 2002. Critical role for STAT4 activation by type I interferons in the interferon- $\gamma$ response to viral infection. Science. 297:2063-2066.

26. Wechsler-Reya, R., and Scott, M.P. 2001. The developmental biology of brain tumors. Annu. Rev. Neurosci. 24:385-428.

27. Ruiz i Altaba, A., Sanchez, P., and Dahmane, N. 2002. Gli and hedgehog in cancer: tumours, embryos and stem cells. Nat. Rev. Cancer 2:361-372

28. Bumcrot, D.A., Takada, R., and McMahon, A.P. 1995. Proteolytic processing yields two secreted forms of sonic hedgehog. Mol. Cell. Biol. 15:2294-2303.

29. Porter, J.A., et al. 1995. The product of hedgehog autoproteolytic cleavage active in local and long-range signalling. Nature. 374:363-366.

30. Huang, M., et al. 2002. Chromatin-remodelling factor BRG1 selectively activates a subset of interferon- $\alpha$-inducible genes. Nat. Cell Biol. 4:774-781.

31. Rogge, L., et al. 1998. The role of Stat 4 in species-specific regulation of Th cell development by type I IFNs. J. Immunol. 161:6567-6574.

32. Wenner, C.A., Guler, M.L., Macatonia, S.E., O'Garra, A., and Murphy, K.M. 1996. Roles of IFN- $\gamma$ and IFN- $\alpha$ in IL-12-induced T helper cell-1 development. J. Immunol. 156:1442-1447.

33. Farrar, J.D., et al. 2000. Selective loss of type I interferon-induced STAT4 activation caused by a minisatellite insertion in mouse Stat 2 . Nat. Immunol. 1:65-69.

34. Bromberg, J. 2002. Stat proteins and oncogenesis. J. Clin. Invest. 109:1139-1142. doi:10.1172/JCI200215617.

35. Schaefer, L.K., Ren, Z., Fuller, G.N., and Schaefer, T.S. 2002. Constitutive activation of Stat3alpha in brain tumors: localization to tumor endothelial cells and activation by the endothelial tyrosine kinase receptor (VEGFR-2). Oncogene. 21:2058-2065.
36. Dahmane, N., and Ruiz i Altaba, A. 1999. Sonic hedgehog regulates the growth and patterning of the cerebellum. Development. 126:3089-3100.

37. Wallace, V.A. 1999. Purkinje-cell-derived Sonic hedgehog regulates granule neuron precursor cell proliferation in the developing mouse cerebellum. Curr. Biol. 9:445-448.

38. Wechsler-Reya, R.J., and Scott, M.P. 1999. Control of neuronal precursor proliferation in the cerebellum by Sonic Hedgehog. Neuron. 22:103-114.

39. Matise, M.P., and Joyner, A.L. 1999. Gli genes in development and cancer. Oncogene. 18:7852-7859.

40. Weiner, H.L., et al. 2002. Induction of medulloblastomas in mice by sonic hedgehog, independent of Gli1. Cancer Res. 62:6385-6389.

41. Corbin, J.G., et al. 1996. Targeted CNS expression of interferon- $\gamma$ in transgenic mice leads to hypomyelination, reactive gliosis, and abnormal cerebellar development. Mol. Cell. Neurosci. 7:354-370.

42. LaFerla, F.M., Sugarman, M.C., Lane, T.E., and Leissring, M.A. 2000. Regional hypomyelination and dysplasia in transgenic mice with astrocyte-targeted expression of interferon- $\gamma$. J. Mol. Neurosci. 15:45-59.

43. Del Valle, L., et al. 2002. Expression of human neurotropic polyomavirus JCV late gene product agnoprotein in human medulloblastoma. J. Natl. Cancer Inst. 94:267-273.

44. Fine, H.A. 2002. Polyomavirus and medulloblastoma: a smoking gun or guilt by association? J. Natl. Cancer Inst. 94:240-241.

45. Cebulla, C.M., Miller, D.M., and Sedmak, D.D. 1999. Viral inhibition of interferon signal transduction. Intervirology. 42:325-330.

46. Hariya, Y., Yokosawa, N., Yonekura, N., Kohama, G., and Fuji, N. 2000. Mumps virus can suppress the effective augmentation of HPC-induced apoptosis by IFN-gamma through disruption of IFN signaling in U3937 cells. Microbiol. Immunol. 44:537-541.

47. Parisien, J.P., Lau, J.F., and Horvath, C.M. 2002. STAT2 acts as a host range determinant for species-specific paramyxovirus interferon antagonism and simian virus 5 replication. J. Virol. 76:6435-6441.

48. Starr, R., and Hilton, D.J. 1999. Negative regulation of the JAK/STAT pathway. Bioessays. 21:47-52.

49. Dupuis, S., et al. 2001. Impairment of mycobacterial but not viral immunity by a germline human STAT1 mutation. Science. 293:300-303.

50. Dupuis, S., et al. 2003. Impaired response to interferon- $\alpha / \beta$ and lethal viral disease in human STAT1 deficiency. Nat. Genet. 33:388-391.

51. Friedman, H.S., Oakes, W.J., Bigner, S.H., Wikstrand, C.J., and Bigner, D.D. 1991. Medulloblastoma: tumor biological and clinical perspectives. J. Neurooncol. 11:1-15.

52. Packer, R.J., Cogen, P., Vezina, G., and Rorke, L.B. 1999. Medulloblastoma: clinical and biologic aspects. Neuro-oncol. 1:232-250. 